\title{
Cytotoxic Isoprenoids from the Red Sea Soft Corals Sarcophyton glaucum and Xenia umbellate
}

\author{
Elham A. Selim , Khalid O. Al-Footy and Seif-Eldin N. Ayyad*, \\ Department of Chemistry, Faculty of Science, King Abdulaziz University, \\ PO. Box 80203, Jeddah 21589, Saudi Arabia \\ *E-mail: snayyad2@yahoo.com
}

\begin{abstract}
Three cytotoxic cembranoids, sarcophytolol (1), sarcophytolide B (2) and sarcophytolide $C$ (3) were identified from the Et2O-soluble fraction derived from the crude extract of the soft coral Sarcophyton glaucum, along with three known aromadendrane-type sesquiterpenoids, aromadendrene (4), palustrol (5) and viridiflorol (6) were isolated from Xenia umbellata collected from the Red Sea water, Jeddah. Chemical structures were determined by analyzing their NMR and MS data. Compounds 1-6 were evaluated for potential cytotoxic activities, employing five cancer panels; HepG2, MCF-7, A549, PC-3 and VERO. Interestingly, compounds 1 and 3 exhibited selective cytotoxic effects on HepG2 and showed no toxicity to the other cell lines. However, 2 showed selective cytotoxic effect on MCF-7 with no toxicity on other cell lines. Meanwhile compounds 4-6 showed cytotoxic activity against both MCF-7 and HepG2 with no toxicity on normal VERO cells.
\end{abstract}

Keywords: Red Sea, soft coral, Alcyoniidae, terpenoids, cytotoxicity

\section{Introduction}

Cancer is one of the major causes of death, with approximately 12.7 million cancer cases, $60 \%$ of them died in 2008 alone ${ }^{[1]}$. This makes cancer drug discovery more urgent and deep needs ${ }^{[2]}$. Natural products 
remain important despite the current trend toward rational drug designs

[3]. Water is covering two-thirds of the earth's surface and home to nearly one million multicellular (plants and animals) and one billion unicellular (Distributed under 100 different phyla) organisms ${ }^{[4]}$. Living in harsh environment makes the marine organisms struggling to survive, which is accruing through chemical defense mechanism. Thus, marine organisms represent a generator for natural products with diversity of chemical structures and biological activities ${ }^{[5]}$. The Red Sea represents the tropical and subtropical climatic region with a variety of the endemic biota. Upto-date $c a$. 180 soft corals species were identified world-wide. The majority of them are native to the Red Sea ${ }^{[5]}$. Soft corals are known for the production of terpenoidal metabolites especially cembrane diterpenes and sesquiterpenes ${ }^{[6]}$. The course of our research program aims at the discovery of bioactive and/or new metabolites from marine organisms. We investigated the constituents of the shallow water Red Sea soft corals Xenia umbellata and Sarcophyton galucum (Family Alcyoniidae, Order Alcyonacea, subclass Octocorallia, class Anthozoa, phylum coelenterata). We have isolated six metabolites (Fig. 1) and their cytotoxicity against five cancer panels; HepG2, MCF-7, A549, PC-3 and VERO were studied.

\section{Materials and Methods}

\subsection{General experimental procedure}

Optical rotations were measured on ATAGO POLAX-L 2 polarimeter. EI/MS analyses were carried out on a Shimadzu-QP 2010. GC/MS analyses were carried out using RTX-1 column (30 m, 0.25mm). The 1D and 2D NMR spectra were recorded on Bruker AVANCE III WM $600 \mathrm{MHz}$ spectrometers and ${ }^{13} \mathrm{C}$ NMR at $150 \mathrm{MHz}$. Chemical shifts are given in $\delta c(\mathrm{ppm})$ relative to TMS as internal standard. 

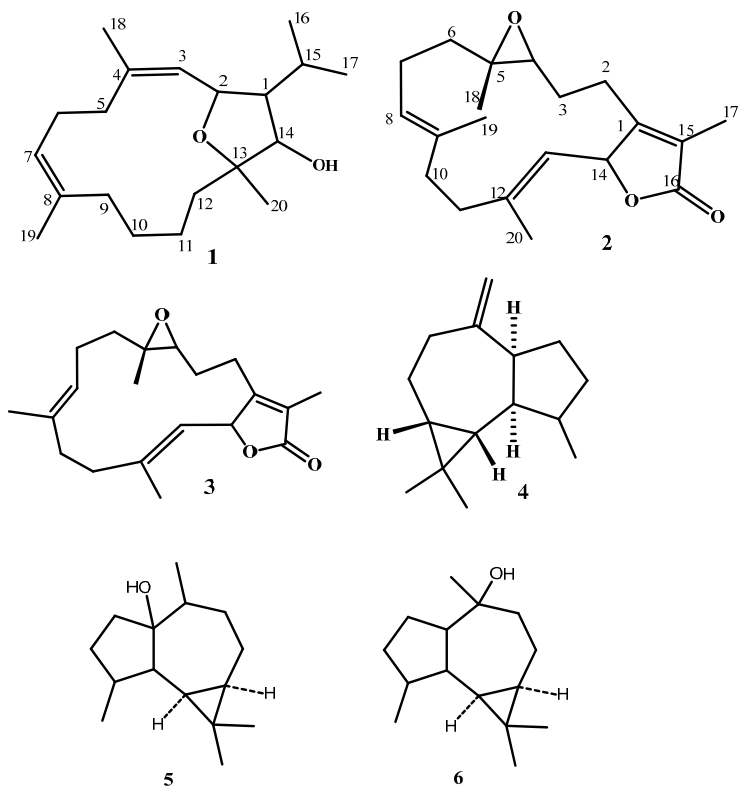

Fig. 1: Compounds isolated from Sarcophyton glaucum (1-3) and Xenia

umbellate (4-6)

Chemical shifts are given in ppm relative to TMS as internal standard. Thin layer chromatography was performed on silica gel (Kieselgel 60, F254) of $0.25 \mathrm{~mm}$ layer thickness. Gel filtration was carried out using Sephadex LH-20. Spots were detected by using ethanol /sulfuric acid as spray reagent.

\subsection{Animal materials}

Soft coral S. glaucum (Order Alcyonacea, Family Alcyoniidae) was collected from the Red Sea coast $\left(21^{\circ} 29^{\prime} 31^{\prime \prime} \mathrm{N} 39^{\circ} 11^{\prime} 24^{\prime \prime} \mathrm{E}\right)$ in Jeddah, at a depth of $5 \mathrm{~m}$, in May, 2012. Collection of this material was immediately subjected to the extraction. A voucher specimen (SC-20128) was deposited in the Faculty of Marine Science, King Abdulaziz University, Jeddah, Saudi Arabia.

The marine invertebrate $X$. umbellata (Alcyonaceae, Xeniidae) had been collected by Snorkeling in the winter on 21 November 2012 along the cost of Red sea in Saudi Arabia, Jeddah (in protected reef slopes Obhor) at a depth of 3-5 m. A voucher specimen (Sx-2012-11) was 
deposited in the Faculty of Marine Science, King Abdulaziz University, Jeddah, Saudi Arabia. The specimens were immediately

frozen until dryness and then extracted.

\subsection{Extraction and isolation}

\section{Sarcophyton gaucum}

The fresh Soft coral $S$. glaucum $(3.0 \mathrm{~kg})$ was minced and extracted by diethyl ether at room temperature $\left(22^{\circ} \mathrm{C}, 3 \mathrm{X} 5\right.$ Liters $)$. The yielded viscous blackish residue ( $20 \mathrm{~g}$ ), was fractionated on NP-Silica employing gradient elution from $n$-hexane to EtOAc, followed by dichloromethane to acetone $(\Phi=50, \mathrm{~L}=100 \mathrm{~cm}, 100 \mathrm{~mL}$ each). Twenty fractions were collected. The fractions were investigated by ${ }^{1} \mathrm{H}-\mathrm{NMR}$ and TLC pattern using $\mathrm{UV}_{254}$ lamp and/or 50\%-sulfuric acid in methanol as spraying reagent. All compounds were purified by PTLC and re-purified by employing Sephadex LH-20 $(\Phi=5, \mathrm{~L}=10 \mathrm{~cm}, 5 \mathrm{~mL}$ each $)$ using a mixture of $\mathrm{MeOH}: \mathrm{CHCl}_{3}$ (9:1). Fraction 5 was purified by RP-18 HPLC $(\mathrm{MeOH} / \mathrm{H} \mathrm{O}, 85: 15)$ to afford yield $\mathbf{1}(8.0 \mathrm{mg})$ and $\mathbf{2}(9.0 \mathrm{mg})$. Fraction 8 was purified by RP-18 HPLC (MeOH/H O, 65:35) to yield 3 (10 mg).

\section{Sarcophytolol, (1)}

Colorless oil $(8.0 \mathrm{mg}, 0.0084 \%)$; $[\alpha]_{\mathrm{D}}^{22}+10.0\left(c 0.02, \mathrm{CHCl}_{3}\right)$; IR $\lambda_{\max }\left(\right.$ film) $\mathrm{cm}^{-1}: 3713,3423,2937,1445,1378,1221,1045 ;{ }^{13} \mathrm{C} \mathrm{NMR}$ $\left(\mathrm{CDCl}_{3}, 150 \mathrm{MHz}\right)$ and ${ }^{1} \mathrm{H} \mathrm{NMR}\left(\mathrm{CDCl}_{3}, 600 \mathrm{MHz}\right)$ spectroscopic data (Table 1); HRESI-MS (positive mode) $\mathrm{m} / z=307.2632 \quad[\mathrm{M}+\mathrm{H}]^{+}$ (Calculated $m / z=306.2559$ for $\mathrm{C}_{20} \mathrm{H}_{34} \mathrm{O}_{2}$ ).

Sarcophytolide B, (2)

Colorless oil (10.0 mg); $[\alpha]_{\mathrm{D}}^{22}-90.0\left(\mathrm{c} 0.02, \mathrm{CHCl}_{3}\right)$; IR $\lambda_{\max }$ (film) $\mathrm{cm}^{-1}: 3472,2925,1748,1454,1392,1090,771 ;{ }^{13} \mathrm{C} \mathrm{NMR}\left(\mathrm{CDCl}_{3}, 150\right.$ $\mathrm{MHz})$ and ${ }^{1} \mathrm{H}$ NMR $\left(\mathrm{CDCl}_{3}, 600 \mathrm{MHz}\right)$ spectroscopic data, (Table 1); HRESI-MS (Positive-ion-mode) $\mathrm{m} / \mathrm{z} 317.2118[\mathrm{M}+\mathrm{H}]^{+}$(Calculated $\mathrm{m} / \mathrm{z}$ $=316.2380$ for $\mathrm{C}_{20} \mathrm{H}_{28} \mathrm{O}_{3}$ ).

\section{Sarcophytolide $C$, (3)}

Colorless oil $(9.0 \mathrm{mg}) ;[\alpha]_{\mathrm{D}}{ }^{22}-60.0\left(\mathrm{c} 0.015, \mathrm{CHCl}_{3}\right) ; \mathrm{IR} \lambda_{\max }$ (film) $\mathrm{cm}^{-1}: 3472,2925,1748,1450,1388,1086,763 ;{ }^{13} \mathrm{C} \mathrm{NMR}\left(\mathrm{CDCl}_{3}, 150\right.$ $\mathrm{MHz})$ and ${ }^{1} \mathrm{H} \mathrm{NMR}\left(\mathrm{CDCl}_{3}, 600 \mathrm{MHz}\right)$ spectroscopic data (Table 1); 
HRESI-MS (Positive-ion-mode) $\mathrm{m} / \mathrm{z} 317.2118[\mathrm{M}+\mathrm{H}]^{+}$(Calculated $\mathrm{m} / \mathrm{z}$ $=316.2380$ for $\mathrm{C}_{20} \mathrm{H}_{28} \mathrm{O}_{3}$ ).

\section{Xenia umbellata}

The residue $(14 \mathrm{~g})$ was homogenized with suitable amount of silica gel and then subjected to Si-gel open column $(500 \mathrm{~g}, 75 \times 2 \mathrm{~cm})$ for separation. $n$-hexane was used as eluent and the polarity was increased by adding increasing amounts of EtOAc. Fractions were collected (50 $\mathrm{ml}$ ). The course of fractionation was traced using TLC technique with the aid of both UV light and spray reagent. For impure fractions preparative TLC (PTLC) was applied to isolate a single compound.

\section{Aromadendrene, (4)}

The fraction eluted with $n$-hexane, $\mathrm{R}_{\mathrm{f}}=0.98(16.1 \mathrm{mg})$ was repurified by PTLC. The violet color band (with spray reagent) $R_{f}=0.90$ gave pale yellow oil $\left(9.3 \mathrm{mg}\right.$ ). IR $v_{\max }$ (neat) $\mathrm{cm}^{-1}: 3080,1635,1460$, 1377, and 895; GC-MS m/z (rel. int.): $204\left(\mathrm{M}^{+}\right), \mathrm{m} / \mathrm{z} 41$ (base peak). ${ }^{1} \mathrm{H}$ NMR $\left(\mathrm{CDCl}_{3}, 600 \mathrm{MHz}\right): \delta=0.24(1 \mathrm{H}, \mathrm{dd}, J=10.9,9.3 \mathrm{~Hz}, \mathrm{H}-6), 0.55$ $(1 \mathrm{H}$, ddd, $J=10.9,9.3,5.9 \mathrm{~Hz}, \mathrm{H}-7), 0.94\left(3 \mathrm{H}, \mathrm{d}, J=6.7 \mathrm{~Hz}, \mathrm{H}_{3}-4\right), 0.96$ $\left(3 \mathrm{H}, \mathrm{s}, \mathrm{H}_{3}-12\right), 1.01\left(3 \mathrm{H}, \mathrm{s}, \mathrm{H}_{3}-13\right) .4 .74\left(1 \mathrm{H}, \mathrm{s}, \mathrm{H}_{\mathrm{a}}-14\right), 4.71\left(1 \mathrm{H}, \mathrm{s}, \mathrm{H}_{\mathrm{b}}-\right.$ 14); ${ }^{13} \mathrm{C}$ NMR $(\mathrm{CDCl} 3,150 \mathrm{MHz}): \delta=152.3$ (s, C-10), $109.8\left(\mathrm{CH}_{2}, \mathrm{C}-\right.$ 14), 50.8 (CH, C-1), 42.2 (CH, C-5), $37.9(\mathrm{CH}, \mathrm{C}-4), 35.8\left(\mathrm{CH}_{2}, \mathrm{C}-9\right)$, $31.3\left(\mathrm{CH}_{2}, \mathrm{C}-3\right), 28.7\left(\mathrm{CH}_{3}, \mathrm{C}-13\right), 28.3\left(\mathrm{CH}_{2}, \mathrm{C}-2\right), 24.9(\mathrm{CH}, \mathrm{C}-7)$, $23.6(\mathrm{CH}, \mathrm{C}-6), 22.20\left(\mathrm{CH}_{2}, \mathrm{C}-8\right), 17.2(\mathrm{C}, \mathrm{C}-11), 16.4\left(\mathrm{CH}_{3}, \mathrm{C}-15\right)$, 15.9 (q, C-12).

\section{Palustrol, (5)}

The fraction eluted by $n$-hexane/ ethylacetate $(9: 1), \mathrm{R}_{\mathrm{f}}=0.80(240.9$ $\mathrm{mg}$ ) was re-purified by PTLC using the solvent system $n$-hexane/ ethylacetate (8:2). The brown color band (with spray reagent) $R_{f}=0.74$ gave colorless oil $\left(124.5 \mathrm{mg}\right.$ ). IR $v_{\max }$ (neat) $\mathrm{cm}^{-1}: 3326,2954,2868$, 1471, 1375, 1264, 992, 940, 890; GCMS m/z (rel. int.): : $222\left(\mathrm{M}^{+}, 2 \%\right)$, 204 (19), 189 (12), 165 (12), 161 (28), 147 (21), 122(75), 111(100), 109(43), 107(48). ${ }^{1} \mathrm{H}$ NMR $\left(\mathrm{CDCl}_{3}, 600 \mathrm{MHz}\right): \delta=1.84\left(1 \mathrm{H}, \mathrm{m}, \mathrm{H}_{\mathrm{a}}-2\right)$, $1.37\left(1 \mathrm{H}, \mathrm{m}, \mathrm{H}_{\mathrm{b}}-2\right), 1.82\left(2 \mathrm{H}, \mathrm{m}, \mathrm{H}_{2}-3\right), 2.15(1 \mathrm{H}, \mathrm{m}, \mathrm{H}-4), 1.60(1 \mathrm{H}, \mathrm{dd}$, $J=10.2,10.2 \mathrm{~Hz}, \mathrm{H}-5), 0.51(1 \mathrm{H}, \mathrm{dd}, J=10.8,9 \mathrm{~Hz}, \mathrm{H}-6), 0.68(1 \mathrm{H}$, ddd, $J=10.8,9,6.6 \mathrm{~Hz}, \mathrm{H}-7), 1.88\left(1 \mathrm{H}, \mathrm{m}, \mathrm{H}_{\mathrm{a}}-8\right), 1.06\left(1 \mathrm{H}, \mathrm{m}, \mathrm{H}_{\mathrm{b}}-8\right)$, $1.47\left(1 \mathrm{H}, \mathrm{m}, \mathrm{H}_{\mathrm{a}}-9\right), 1.35\left(1 \mathrm{H}, \mathrm{m}, \mathrm{H}_{\mathrm{b}}-9\right), 1.27(1 \mathrm{H}, \mathrm{m}, \mathrm{H}-10), 1.03(3 \mathrm{H}, \mathrm{s}$, $\left.\mathrm{H}_{3}-12\right), 1.06\left(3 \mathrm{H}, \mathrm{s}, \mathrm{H}_{3}-13\right), 0.98\left(3 \mathrm{H}, \mathrm{d}, J=6.6 \mathrm{~Hz}, \mathrm{H}_{3}-14\right), 0.92(3 \mathrm{H}, \mathrm{d}$, 
$\left.J=6.6 \mathrm{~Hz}, \mathrm{H}_{3}-15\right) ;{ }^{13} \mathrm{C} \mathrm{NMR}\left(\mathrm{CDCl}_{3}, 150 \mathrm{MHz}\right): \delta=85.7(\mathrm{CH}, \mathrm{C}-1)$, 47.2 (CH, C-5), 46.1 (CH, C-10), $38.9\left(\mathrm{CH}_{2}, \mathrm{C}-2\right), 35.0(\mathrm{CH}, \mathrm{C}-4), 32.8$ $\left(\mathrm{CH}_{2}, \mathrm{C}-3\right), 32.3\left(\mathrm{CH}_{2}, \mathrm{C}-9\right), 28.9\left(\mathrm{CH}_{3}, \mathrm{C}-13\right), 27.1(\mathrm{CH}, \mathrm{C}-7), 24.5$ $\left(\mathrm{CH}_{2}, \mathrm{C}-8\right), 22.0(\mathrm{CH}, \mathrm{C}-6), 19.9(\mathrm{C}, \mathrm{C}-11), 18.7\left(\mathrm{CH}_{3}, \mathrm{C}-14\right), 17.7$ $\left(\mathrm{CH}_{3}, \mathrm{C}-15\right), 15.6\left(\mathrm{CH}_{3}, \mathrm{C}-12\right)$.

\section{Viridiflorol, (6)}

The fraction eluted by $n$-hexane/ ethylacetate $(9: 1), \mathrm{R}_{\mathrm{f}}=0.49(240.9$ mg) was re-purified by PTLC using the solvent system $n$-hexane/ ethylacetate (8:2). The brown color band (with spray reagent) $\mathrm{R}_{\mathrm{f}}=0.56$ gave white crystalline material $(22.7 \mathrm{mg})$. IR $v_{\max }$ (neat) $\mathrm{cm}^{-1}: 3350$, 2925, 2866, 1466, 1376, 1112, 990, 941, 887, 682; GCMS m/z (rel. int.): $204\left[\mathrm{M}^{+}\right]$(7), 189 (6), 161 (19), 147 (14), 133 (10), 122 (44), 109 (53), 95 (30), 81 (36), 69 ,(44), 55 (28), 43 (100); ${ }^{1} \mathrm{H} \mathrm{NMR}=\left(\mathrm{CDCl}_{3}\right.$, $600 \mathrm{MHz}): \delta=0.33(\mathrm{dd}, J=10.2,9.0 \mathrm{~Hz}, \mathrm{H}-6), 0.73$ (ddd, $J=10.8,9.0,6$ $\mathrm{Hz}, \mathrm{H}-7), 0.94\left(3 \mathrm{H}, \mathrm{d}, J=7.2 \mathrm{~Hz}, \mathrm{H}_{3}-15\right), 0.98\left(3 \mathrm{H}, \mathrm{s}, \mathrm{H}_{3}-12\right), 1.04(3 \mathrm{H}$, $\left.\mathrm{s}, \mathrm{H}_{3}-13\right), 1.14\left(3 \mathrm{H}, \mathrm{s}, \mathrm{H}_{3}-14\right) ;{ }^{13} \mathrm{C} \mathrm{NMR}(\mathrm{CDCl} 3,150 \mathrm{MHz}): \delta=74.6(\mathrm{C}$, $\mathrm{C}-10), 53.8$ (CH, C-1), 40.8( $\left.\mathrm{CH}_{2}, \mathrm{C}-9\right), 39.2(\mathrm{CH}, \mathrm{C}-5), 38.4(\mathrm{CH}, \mathrm{C}-4)$, $30.8\left(\mathrm{CH}_{2}, \mathrm{C}-3\right), 30.5\left(\mathrm{CH}_{3}, \mathrm{C}-14\right), 28.7\left(\mathrm{CH}_{2}, \mathrm{C}-8\right), 25.0\left(\mathrm{CH}_{2}, \mathrm{C}-2\right)$, $24.6(\mathrm{CH}, \mathrm{C}-6), 23.4\left(\mathrm{CH}_{3}, \mathrm{C}-12\right), 20.3(\mathrm{CH}, \mathrm{C}-7), 19.20(\mathrm{CH}, \mathrm{C}-11)$, $16.0\left(\mathrm{CH}_{3}, \mathrm{C}-13\right), 15.4\left(\mathrm{CH}_{3}, \mathrm{C}-15\right)$.

\subsection{Cell cytotoxicity assay}

\section{Cell culture:}

HepG2, MCF-7, A549 and PC-3 cell lines were grown in RPMI-1640. VERO and A549 cells were grown in DMEM. All culture media were supplemented with $10 \%$ heat inactivated FBS, 100 units $/ \mathrm{ml}$ of penicillin and $100 \mathrm{mg} / \mathrm{mL}$ of streptomycin and maintained at $37{ }^{\circ} \mathrm{C}$ in a humidified atmosphere containing $5 \% \mathrm{CO}_{2}$. The cells were maintained as "monolayer culture" by serial subculturing.

\section{MTT Cytotoxicity assay:}

The cytotoxic activity of the compounds was tested with five different mammalian cell lines HepG2, MCF-7, A549, PC-3 and VERO. The cell lines were obtained from American Type Culture Collection (ATCC) (Mosmann, 1983). The cells were cultivated at $37^{\circ} \mathrm{C}$ and $10 \%$ $\mathrm{CO}_{2}$ in RPMI-1640 (Lonza, 12-702F) medium supplemented with $10 \%$ fetal bovine serum (FBS, Lonza, Cat. No.14-801E), $100 \mathrm{IU} / \mathrm{ml}$ pencillin 
and $100 \mu \mathrm{g} / \mathrm{ml}$ streptomycin (Lonza, 17-602E). Inhibition of proliferation was measured in 96-well plate. $60 \mu \mathrm{L}$ of serial dilutions of the test compounds were given to $120 \mu \mathrm{L}$ of the suspended cells $(50,000 / \mathrm{mL})$ in wells of 96-well plates. After 5 days of growth each well was judged visually under the microscope (Mosman, 1983). The metabolic activity of the cells was measured by an MTT (3-(4,5Dimethyl-2-thiazolyl)-2,5-diphenyl-2H-tetrazolium bromide) assay ERVA, Cat. No. 20395.01) was added to each well and incubated for 4 hours at $37^{\circ} \mathrm{C}$ and $10 \% \mathrm{CO}_{2}$. After that the medium was removed and formazan particles were dissolved by adding $100 \mu \mathrm{l}$ Isopropanol $/ \mathrm{HCl}$ solution. The plates were then incubated for $15 \mathrm{~min}$ on shaker $(600$ r.p.m/ min) to dissolve the formazan crystals. The intensity of the purple color was measured at $\lambda 540 \mathrm{~nm}$ by microplate reader (ELx800 Absorbance Microplate Reader, BioTek). The cytotoxicity was recorded at the concentrations that gave $50 \%$ growth inhibition $\left(\mathrm{IC}_{50}\right)$.

\section{Results and Discussion}

Compound 1 was isolated as a faint yellow oil $(8.0 \mathrm{mg})$ with $[\alpha]_{\mathrm{D}}{ }^{22}$ $+10.0\left(c 0.02, \mathrm{CHCl}_{3}\right)$. The structure elucidation commenced when the molecular formula of $\mathbf{1}, \mathrm{C}_{20} \mathrm{H}_{34} \mathrm{O}_{2}$ was established by LCESI-MS (negative mode) $m / z=305.2[\mathrm{M}-\mathrm{H}]^{+}, 287.1\left[\mathrm{M}-\mathrm{H}_{2} \mathrm{O}\right]^{+}, 269.1\left[\mathrm{M}-2 \mathrm{H}_{2} \mathrm{O}\right]^{+}$. This result was validated by HRESI-MS (positive mode) $\mathrm{m} / z=307.2632$ $[\mathrm{M}+\mathrm{H}]]^{+}$. The ${ }^{13} \mathrm{C}\left({ }^{1} \mathrm{H}\right.$ decoupled $)$ and DEPT NMR spectra of $\mathbf{1}$, showed 20 resonances attributable to five methyl, six methylenes, six methines and three quaternary carbons (Table 1).

Two of the four elements of unsaturation, as indicated by the molecular formula of $\mathbf{1}$, are attributed to two $\mathrm{C}=\mathrm{C}$ double bonds (Table 1); thus, the molecule is bicyclic. As the ${ }^{1} \mathrm{H}$ and ${ }^{13} \mathrm{C}$ NMR data enabled all but one of the hydrogen atoms within 1 to be accounted for, it was evident that the remaining proton was present as part of a hydroxyl. function, a deduction supported by the $\mathrm{OH}$ stretching in IR spectral data $\left(\lambda_{\max } 3423 \mathrm{~cm}^{-1}\right)$. After association of all protons with directly bonded carbons via HSQC spectral measurement, it was possible to deduce the planar structure of $\mathbf{1}$ by interpretation of the ${ }^{1} \mathrm{H}-{ }^{1} \mathrm{H}$ COESY and ${ }^{1} \mathrm{H}-{ }^{13} \mathrm{C}$ HMBC spectra. 
Table 1. ${ }^{1} \mathrm{H}\left[\mathrm{CDCl}_{3}, 600 \mathrm{MHz}\right]$ and ${ }^{13} \mathrm{C} \mathrm{NMR}\left[\mathrm{CDCl}_{3}, 150 \mathrm{MHz}\right] \mathrm{NMR}$ spectral data of $\mathbf{1 - 3}{ }^{\mathrm{a}}$

\begin{tabular}{|c|c|c|c|c|c|c|}
\hline \multicolumn{3}{|r|}{1} & \multicolumn{2}{|r|}{2} & \multicolumn{2}{|r|}{3} \\
\hline & $\delta^{13} C^{b}$ & $\delta^{1} \mathbf{H}^{\mathrm{c}}$ & $\delta^{13} \mathrm{C}$ & $\delta^{1} \mathbf{H}$ & $\delta^{13} \mathrm{C}^{\mathrm{b}}$ & $\delta^{1} \mathbf{H}$ \\
\hline 1 & $46.6(d)$ & $1.32(1 \mathrm{H}, \mathrm{m})$ & $162.2(\mathrm{~s})$ & - & $162.9(\mathrm{~s})$ & - \\
\hline 2 & $71.1(\mathrm{~d})$ & $4.53(1 \mathrm{H}, \mathrm{dd}, 10.2,5.4)$ & $37.4(t)$ & $\begin{array}{l}2.40(2 \mathrm{H}, \mathrm{ddd}, 10.8, \\
4.2,3.0)\end{array}$ & $36.9(\mathrm{t})$ & $\begin{array}{l}2.40(2 \mathrm{H}, \text { ddd, } 10.8 \\
4.2,3.0)\end{array}$ \\
\hline 3 & $\begin{array}{l}125.4 \\
\text { (d) }\end{array}$ & $5.27(1 \mathrm{H}, \mathrm{d}, 10.2)$ & $25.2(\mathrm{t})$ & $\begin{array}{l}1.90(1 \mathrm{H}, \mathrm{m}) \\
1.70(1 \mathrm{H}, \mathrm{m})\end{array}$ & $25.8(\mathrm{t})$ & $\begin{array}{l}1.90, \mathrm{~m} \\
1.70, \mathrm{~m} \\
\end{array}$ \\
\hline 4 & $\begin{array}{l}138.6 \\
(\mathrm{~s}) \\
\end{array}$ & - & $61.4(\mathrm{~d})$ & $2.68(1 \mathrm{H}, \mathrm{dd}, 4.8,4.2)$ & $61.8(\mathrm{~d})$ & $\begin{array}{l}2.66(1 \mathrm{H}, \mathrm{dd}, 6.6 \text {, } \\
6.0)\end{array}$ \\
\hline 5 & $39.9(\mathrm{t})$ & $\begin{array}{l}2.19(1 \mathrm{H}, \mathrm{m}) \\
1.97(1 \mathrm{H}, \mathrm{m})\end{array}$ & $59.9(\mathrm{~s})$ & - & $60.2(\mathrm{~s})$ & - \\
\hline 6 & $25.2(\mathrm{t})$ & $\begin{array}{l}2.32(1 \mathrm{H}, \mathrm{m}) \\
2.11(1 \mathrm{H}, \mathrm{m})\end{array}$ & $39.0(\mathrm{t})$ & $1.10(2 \mathrm{H}, \mathrm{m})$ & $38.4(t)$ & $1.10(2 \mathrm{H}, \mathrm{m})$ \\
\hline 7 & $\begin{array}{l}124.1 \\
(\mathrm{~d})\end{array}$ & $5.00(1 \mathrm{H}, \mathrm{dd}, 10.2,5.4)$ & $23.3(\mathrm{t})$ & $2.13(2 \mathrm{H}, \mathrm{m})$ & 23. $0(\mathrm{~d})$ & $2.13(2 \mathrm{H}, \mathrm{m})$ \\
\hline 8 & $\begin{array}{l}136.0 \\
(\mathrm{~s})\end{array}$ & - & $124.9(\mathrm{~d})$ & $5.17(1 \mathrm{H}, \mathrm{dd}, 9.6,5.4)$ & $\begin{array}{c}124.6 \\
(\mathrm{~d})\end{array}$ & $\begin{array}{l}5.11(1 \mathrm{H}, \quad \mathrm{dd}, 8.4, \\
7.8)\end{array}$ \\
\hline 9 & $33.7(t)$ & $\begin{array}{l}2.40(1 \mathrm{H}, \mathrm{m}) \\
1.29(1 \mathrm{H}, \mathrm{m})\end{array}$ & $144.0(\mathrm{~s})$ & - & 145.(s) & - \\
\hline 10 & $18.7(\mathrm{t})$ & $\begin{array}{l}1.77(1 \mathrm{H}, \mathrm{m}) \\
1.29(1 \mathrm{H}, \mathrm{m})\end{array}$ & $36.4(\mathrm{t})$ & $\begin{array}{l}2.01(1 \mathrm{H}, \text { ddd, } 13.2, \\
10.8,2.4) \\
2.20(1 \mathrm{H}, \quad \text { ddd, } 18.0, \\
12.6,9.0)\end{array}$ & $35.1(\mathrm{t})$ & $\begin{array}{l}2.05(1 \mathrm{H}, \mathrm{m}) \\
2.20(1 \mathrm{H}, \mathrm{m})\end{array}$ \\
\hline 11 & $31.9(\mathrm{t})$ & $\begin{array}{l}1.77(1 \mathrm{H}, \mathrm{m}) \\
1.28(1 \mathrm{H}, \mathrm{m})\end{array}$ & $27.6(t)$ & $\begin{array}{l}2.10(1 \mathrm{H}, \quad \text { ddd, } 13.2, \\
5.4,2.4) \\
2,78,(1 \mathrm{H}, \quad \text { ddd, } 18.0, \\
9.6,7.8)\end{array}$ & $26.8(t)$ & $\begin{array}{l}2.10(1 \mathrm{H}, \mathrm{m}) \\
2,69(1 \mathrm{H}, \mathrm{m})\end{array}$ \\
\hline 12 & $35.2(\mathrm{t})$ & $\begin{array}{l}2.13(1 \mathrm{H}, \mathrm{m}) \\
2.00(1 \mathrm{H}, \mathrm{m})\end{array}$ & $135.6(\mathrm{~s})$ & - & $134.5(\mathrm{~s})$ & - \\
\hline 13 & $75.0(\mathrm{~s})$ & - & $121.0(\mathrm{~d})$ & $\begin{array}{l}5.05(1 \mathrm{H}, \mathrm{dd}, 10.2, \\
1.2)\end{array}$ & $123.1(\mathrm{~s})$ & $\begin{array}{l}4.99(1 \mathrm{H}, \mathrm{dd}, 9.6 \text {, } \\
1.2)\end{array}$ \\
\hline 14 & $71.9(\mathrm{~d})$ & $3.88(1 \mathrm{H}, \mathrm{d}, 9.6)$ & $78.8(\mathrm{~d})$ & $\begin{array}{l}5.57(1 \mathrm{H}, \quad \mathrm{dd}, 10.2 \\
1.2)\end{array}$ & 79.9 (d) & $\begin{array}{l}5.45(1 \mathrm{H}, \mathrm{dd}, 9.6 \text {, } \\
1.2)\end{array}$ \\
\hline 15 & $29.0(\mathrm{~d})$ & $1.18(1 \mathrm{H}, \mathrm{m})$ & $120.6(\mathrm{~s})$ & - & $119.1(\mathrm{~s})$ & - \\
\hline 16 & $20.8(q)$ & $0.88(3 \mathrm{H}, \mathrm{d}, 6.6)$ & $174.0(\mathrm{~s})$ & - & $175.0(\mathrm{~s})$ & - \\
\hline 17 & $20.3(\mathrm{q})$ & $0.73(3 \mathrm{H}, 6.6)$ & $15.4(\mathrm{q})$ & $1.65(3 \mathrm{H}, \mathrm{s})$ & $15.5(\mathrm{q})$ & $1.60(3 \mathrm{H}, \mathrm{s})$ \\
\hline 18 & $24.2(\mathrm{q})$ & $1.65(3 \mathrm{H}, \mathrm{s})$ & $17.1(\mathrm{q})$ & $1.28(3 \mathrm{H}, \mathrm{s})$ & $18.1(\mathrm{q})$ & $1.27(3 \mathrm{H}, \mathrm{s})$ \\
\hline 19 & $17.3(\mathrm{q})$ & $1.61(3 \mathrm{H}, \mathrm{s})$ & $16.1(\mathrm{q})$ & $1.91(3 \mathrm{H}, \mathrm{s})$ & $16.7(q)$ & $1.93(3 \mathrm{H}, \mathrm{s})$ \\
\hline 20 & $15.1(q)$ & $1.02(3 \mathrm{H}, \mathrm{s})$ & 8.92 & $1.88(3 \mathrm{H}, \mathrm{s})$ & $9.2(q)$ & $1.86(3 \mathrm{H}, \mathrm{s})$ \\
\hline
\end{tabular}




\begin{tabular}{|c|c|c|c|c|c|c|}
\hline & & 1 & \multicolumn{2}{|r|}{2} & \multicolumn{2}{|r|}{3} \\
\hline & $\delta^{13} \mathbf{C}^{\mathrm{b}}$ & $\delta^{1} \mathbf{H}^{\mathrm{c}}$ & $\delta^{13} \mathrm{C}$ & $\delta^{1} \mathbf{H}$ & $\delta^{13} \mathrm{C}^{\mathrm{b}}$ & $\delta^{1} \mathbf{H}$ \\
\hline 1 & $\begin{array}{l}46.6 \\
(d)\end{array}$ & $1.32(1 \mathrm{H}, \mathrm{m})$ & $\begin{array}{c}162.2 \\
(\mathrm{~s})\end{array}$ & - & $\begin{array}{c}162.9 \\
(\mathrm{~s})\end{array}$ & - \\
\hline 2 & $\begin{array}{l}71.1 \\
\text { (d) }\end{array}$ & 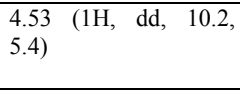 & $\begin{array}{c}37.4 \\
(\mathrm{t})\end{array}$ & $\begin{array}{ll}2.40 \quad(2 \mathrm{H}, \\
\text { ddd, } 10.8, \\
4.2,3.0)\end{array}$ & $36.9(t)$ & $2.40(2 \mathrm{H}, \mathrm{ddd}, 10.8,4.2,3.0)$ \\
\hline 3 & $\begin{array}{l}125.4 \\
\text { (d) }\end{array}$ & $5.27(1 \mathrm{H}, \mathrm{d}, 10.2)$ & $\begin{array}{c}25.2 \\
(\mathrm{t})\end{array}$ & $\begin{array}{ll}1.90 & (1 \mathrm{H}, \\
\mathrm{m}) & \\
1.70 & (1 \mathrm{H}, \\
\mathrm{m}) & \\
\end{array}$ & $25.8(\mathrm{t})$ & $\begin{array}{l}1.90, \mathrm{~m} \\
1.70, \mathrm{~m}\end{array}$ \\
\hline 4 & $\begin{array}{l}138.6 \\
(\mathrm{~s})\end{array}$ & - & $\begin{array}{l}61.4 \\
\text { (d) }\end{array}$ & $\begin{array}{ll}2.68 & (1 \mathrm{H}, \\
\mathrm{dd}, & 4.8, \\
4.2) & \\
\end{array}$ & $61.8(\mathrm{~d})$ & $2.66(1 \mathrm{H}, \mathrm{dd}, 6.6,6.0)$ \\
\hline 5 & $\begin{array}{l}39.9 \\
(\mathrm{t})\end{array}$ & $\begin{array}{l}2.19(1 \mathrm{H}, \mathrm{m}) \\
1.97(1 \mathrm{H}, \mathrm{m})\end{array}$ & $\begin{array}{c}59.9 \\
(\mathrm{~s})\end{array}$ & - & $60.2(\mathrm{~s})$ & - \\
\hline 6 & $\begin{array}{l}25.2 \\
(\mathrm{t})\end{array}$ & $\begin{array}{l}2.32(1 \mathrm{H}, \mathrm{m}) \\
2.11(1 \mathrm{H}, \mathrm{m})\end{array}$ & $\begin{array}{c}39.0 \\
(\mathrm{t})\end{array}$ & $\begin{array}{l}1.10 \quad(2 \mathrm{H}, \\
\mathrm{m})\end{array}$ & $38.4(t)$ & $1.10(2 \mathrm{H}, \mathrm{m})$ \\
\hline 7 & $\begin{array}{l}124.1 \\
\text { (d) }\end{array}$ & $\begin{array}{l}5.00 \quad(1 \mathrm{H}, \mathrm{dd}, \quad 10.2 \text {, } \\
5.4)\end{array}$ & $\begin{array}{c}23.3 \\
(\mathrm{t})\end{array}$ & $\begin{array}{ll}2.13 & (2 \mathrm{H}, \\
\mathrm{m}) & \\
\end{array}$ & 23. $0(\mathrm{~d})$ & $2.13(2 \mathrm{H}, \mathrm{m})$ \\
\hline 8 & $\begin{array}{l}136.0 \\
(\mathrm{~s})\end{array}$ & - & $\begin{array}{c}124.9 \\
\text { (d) }\end{array}$ & $\begin{array}{ll}5.17 & (1 \mathrm{H}, \\
\mathrm{dd}, & 9.6, \\
5.4) & \\
\end{array}$ & $\begin{array}{l}124.6 \\
\text { (d) }\end{array}$ & $5.11(1 \mathrm{H}, \mathrm{dd}, 8.4,7.8)$ \\
\hline 9 & $\begin{array}{l}33.7 \\
(\mathrm{t})\end{array}$ & $\begin{array}{l}2.40(1 \mathrm{H}, \mathrm{m}) \\
1.29(1 \mathrm{H}, \mathrm{m}) \\
\end{array}$ & $\begin{array}{c}144.0 \\
(\mathrm{~s})\end{array}$ & - & 145.(s) & - \\
\hline 10 & $\begin{array}{l}18.7 \\
(\mathrm{t})\end{array}$ & $\begin{array}{l}1.77(1 \mathrm{H}, \mathrm{m}) \\
1.29(1 \mathrm{H}, \mathrm{m})\end{array}$ & $\begin{array}{c}36.4 \\
(\mathrm{t})\end{array}$ & $\begin{array}{lc}2.01 & (1 \mathrm{H}, \\
\text { ddd, } & 13.2, \\
10.8, & 2.4) \\
2.20 & (1 \mathrm{H}, \\
\text { ddd, } & 18.0, \\
12.6,9.0)\end{array}$ & $35.1(\mathrm{t})$ & $\begin{array}{l}2.05(1 \mathrm{H}, \mathrm{m}) \\
2.20(1 \mathrm{H}, \mathrm{m})\end{array}$ \\
\hline 11 & $\begin{array}{l}31.9 \\
(\mathrm{t})\end{array}$ & $\begin{array}{l}1.77(1 \mathrm{H}, \mathrm{m}) \\
1.28(1 \mathrm{H}, \mathrm{m})\end{array}$ & $\begin{array}{c}27.6 \\
(t)\end{array}$ & $\begin{array}{l}2.10 \quad(1 \mathrm{H}, \\
\text { ddd, } 13.2, \\
5.4,2.4) \\
2,78,(1 \mathrm{H}, \\
\text { ddd, } 18.0, \\
9.6,7.8)\end{array}$ & $26.8(t)$ & $\begin{array}{l}2.10(1 \mathrm{H}, \mathrm{m}) \\
2,69(1 \mathrm{H}, \mathrm{m})\end{array}$ \\
\hline 12 & $\begin{array}{l}35.2 \\
(\mathrm{t})\end{array}$ & $\begin{array}{l}2.13(1 \mathrm{H}, \mathrm{m}) \\
2.00(1 \mathrm{H}, \mathrm{m}) \\
\end{array}$ & $\begin{array}{c}135.6 \\
(\mathrm{~s})\end{array}$ & - & $\begin{array}{c}134.5 \\
(\mathrm{~s})\end{array}$ & - \\
\hline 13 & $\begin{array}{l}75.0 \\
(\mathrm{~s})\end{array}$ & - & $\begin{array}{l}121.0 \\
\text { (d) }\end{array}$ & $\begin{array}{ll}5.05 & (1 \mathrm{H}, \\
\mathrm{dd}, & 10.2, \\
1.2) & \\
\end{array}$ & $123.1(\mathrm{~s})$ & $4.99(1 \mathrm{H}, \mathrm{dd}, 9.6,1.2)$ \\
\hline 14 & $\begin{array}{l}71.9 \\
\text { (d) }\end{array}$ & $3.88(1 \mathrm{H}, \mathrm{d}, 9.6)$ & $\begin{array}{c}78.8 \\
\text { (d) }\end{array}$ & $\begin{array}{ll}5.57 & (1 \mathrm{H}, \\
\mathrm{dd}, & 10.2, \\
1.2) & \\
\end{array}$ & 79.9 (d) & $5.45(1 \mathrm{H}, \mathrm{dd}, 9.6,1.2)$ \\
\hline 15 & $\begin{array}{l}29.0 \\
(d)\end{array}$ & $1.18(1 \mathrm{H}, \mathrm{m})$ & $\begin{array}{c}120.6 \\
(\mathrm{~s})\end{array}$ & - & $\begin{array}{c}119.1 \\
(\mathrm{~s})\end{array}$ & - \\
\hline 16 & $\begin{array}{l}20.8 \\
(\mathrm{q})\end{array}$ & $0.88(3 \mathrm{H}, \mathrm{d}, 6.6)$ & $\begin{array}{c}174.0 \\
(\mathrm{~s})\end{array}$ & - & $\begin{array}{c}175.0 \\
(\mathrm{~s})\end{array}$ & - \\
\hline 17 & $\begin{array}{l}20.3 \\
(\mathrm{q})\end{array}$ & $0.73(3 \mathrm{H}, 6.6)$ & $\begin{array}{c}15.4 \\
(\mathrm{q})\end{array}$ & $\begin{array}{l}1.65 \quad(3 \mathrm{H}, \\
\mathrm{s})\end{array}$ & $15.5(\mathrm{q})$ & $1.60(3 \mathrm{H}, \mathrm{s})$ \\
\hline 18 & $\begin{array}{l}24.2 \\
(q)\end{array}$ & $1.65(3 \mathrm{H}, \mathrm{s})$ & $\begin{array}{c}17.1 \\
(\mathrm{q})\end{array}$ & $\begin{array}{l}1.28 \quad(3 \mathrm{H}, \\
\mathrm{s})\end{array}$ & $18.1(\mathrm{q})$ & $1.27(3 \mathrm{H}, \mathrm{s})$ \\
\hline 19 & $\begin{array}{l}17.3 \\
(\mathrm{q})\end{array}$ & $1.61(3 \mathrm{H}, \mathrm{s})$ & $\begin{array}{c}16.1 \\
(q)\end{array}$ & $\begin{array}{l}1.91 \quad(3 \mathrm{H}, \\
\mathrm{s})\end{array}$ & $16.7(q)$ & $1.93(3 \mathrm{H}, \mathrm{s})$ \\
\hline 20 & $\begin{array}{l}15.1 \\
(\mathrm{q}) \\
\end{array}$ & $1.02(3 \mathrm{H}, \mathrm{s})$ & 8.92 & $\begin{array}{ll}1.88 \quad(3 \mathrm{H}, \\
\mathrm{s})\end{array}$ & $9.2(\mathrm{q})$ & $1.86(3 \mathrm{H}, \mathrm{s})$ \\
\hline
\end{tabular}

${ }^{a}$ All assignments are based on 1D and 2D measurements (HMBC, HSQC, COESY). ${ }^{\mathrm{b}}$ Implied multiplicities were determined by DEPT $\left(\mathrm{C}=\mathrm{s}, \mathrm{CH}=\mathrm{d}, \mathrm{CH}_{2}=\mathrm{t}\right) .{ }^{\mathrm{c}} \mathrm{J}$ in $\mathrm{Hz}$. 
From the ${ }^{1} \mathrm{H}-{ }^{1} \mathrm{H}$ COESY spectrum of $\mathbf{1}$, the coupling between $\mathrm{H}_{3}-16$ resonating at $\delta_{\mathrm{H}} 0.88(\mathrm{~d}, J=6.6)$ and $\mathrm{H}-15$ at $\delta_{\mathrm{H}} 1.18(\mathrm{~m})$, as well as this signal and the $\mathrm{H}_{3}-17$ at $\delta_{\mathrm{H}} 0.73(\mathrm{~d}, 6.6)$ indicated the presence of isopropyl moiety. The presence of this moiety and its connection to $\mathrm{C}-1$ were established by long-range $\mathrm{C}-\mathrm{H}$ correlations observed between the resonances of $\mathrm{H}_{3}-16$ and those of $\mathrm{C}-1\left(\delta_{\mathrm{c}} 46.6\right), \mathrm{C}-15\left(\delta_{\mathrm{c}} 29.0\right)$, and $\mathrm{C}-17$ ( $\delta_{c}$ 20.3), between $\mathrm{H}_{3}-17$ and $\mathrm{C}-1, \mathrm{C}-15$, and $\mathrm{C}-16$ ( $\left.\delta_{\mathrm{c}} 20.8\right)$. The existence of a furan ring in which $\mathrm{C}-1$ is part, was concluded by studying the correlations observed between $\mathrm{H}-1$ resonating at $\delta_{\mathrm{H}}(1.32, \mathrm{~m})$ and both $\mathrm{H}-2$ at $\delta_{\mathrm{H}} 4.53(\mathrm{dd}, 10.2,5.4)$ and $\mathrm{H}-14$ at $\delta_{\mathrm{H}} 3.88(\mathrm{~d}, 9.6)$, in addition to the long-range correlations between $\mathrm{H}-1$ with $\mathrm{C}-2\left(\delta_{\mathrm{c}} 71.1\right)$, C-3 $\left(\delta_{\mathrm{c}} 125.4\right), \mathrm{C}-13\left(\delta_{\mathrm{c}} 75.0\right), \mathrm{C}-14\left(\delta_{\mathrm{c}} 71.9\right), \mathrm{C}-15\left(\delta_{\mathrm{c}} 29.0\right), \mathrm{C}-16\left(\delta_{\mathrm{c}}\right.$ $20.8)$ and $\mathrm{C}-17\left(\delta_{\mathrm{c}} 20.3\right)$. Furthermore, HMBC correlation between $\mathrm{H}-2$ and $\mathrm{C}-1\left(\delta_{\mathrm{c}} 46.6\right), \mathrm{C}-2\left(\delta_{\mathrm{c}} 71.1\right), \mathrm{C}-13\left(\delta_{\mathrm{c}} 75.0\right)$ and $\mathrm{C}-14\left(\delta_{\mathrm{c}} 71.9\right)$; also correlation between $\mathrm{H}_{3}-20\left(\delta_{\mathrm{H}} 1.02, \mathrm{~s}\right)$ and $\mathrm{C}-13$ and $\mathrm{C}-14$ indicated the connection between $\mathrm{C}-13$ and $\mathrm{C}-14$ and positioning of $\mathrm{CH}_{3}-20$ on $\mathrm{C}-13$, thus the five membered ring was closed by ether link between $\mathrm{C}-2$ and $\mathrm{C}-13$. On these bases the positioning of the isopropyl group to be attached to $\mathrm{C}-1$ and then the furan ring was established. The presence of a furan ring, in addition to two carbon-carbon double bonds together with the absence of any other unsaturation imply the existence of one more ring. The nature of the second ring was proved to be 14-membered ring based on the following: a) ${ }^{13} \mathrm{C}$ NMR signals $\delta_{\mathrm{c}} 125.4(\mathrm{C}-3), 138.6$ (C-4), 124.1 (C-7) and 136.0 (C-8) indicated the presence of two trisubstituted double bonds without conjugation, a deduction supported by absence of characteristic absorption in UV spectrum, b) ${ }^{1} \mathrm{H}-{ }^{1} \mathrm{H}$ COESY spectral data indicated the possibility to establish three partial structures of consecutive proton systems extending from $\mathrm{H}_{2}-5\left(\delta_{\mathrm{H}} 1.97\right.$, $\mathrm{m}$ and $2.19, \mathrm{~m})$ to $\mathrm{H}_{2}-7\left(\delta_{\mathrm{H}} 5.00, \mathrm{dd}, 10.2,5.4\right)$ through $\mathrm{H}_{2}-6\left(\delta_{\mathrm{H}} 2.11, \mathrm{~m}\right.$ and $2.32, \mathrm{~m})$; from $\mathrm{H}_{2}-9\left(\delta_{\mathrm{H}} 2.4, \mathrm{~m}\right.$ and $\left.1.29, \mathrm{~m}\right)$ to $\mathrm{H}_{2}-12\left(\delta_{\mathrm{H}} 2.13, \mathrm{~m}\right.$, and $2.00, \mathrm{~m})$ through $\mathrm{H}_{2}-10\left(\delta_{\mathrm{H}} 1.29, \mathrm{~m}\right.$ and $\left.1.77, \mathrm{~m}\right)$ and $\mathrm{H}_{2}-11\left(\delta_{\mathrm{H}}\right.$ $1.28, \mathrm{~m}$ and $1.77, \mathrm{~m})$, and finally from $\mathrm{H}-1$ to $\mathrm{H}-3\left(\delta_{\mathrm{H}} 5.27, \mathrm{~d}, 10.2\right)$ through $\mathrm{H}-2, \mathrm{c})$ the connectivities of these partial structures were further established by interpretation of the HMBC correlations (Fig. 2) between the carbons of the three parts as observed from $\mathrm{H}_{3}-16$ to $\mathrm{C}-15, \mathrm{C}-17$, C1, C-2, C-3, C-4 ; from $\mathrm{H}_{3}-18\left(\delta_{\mathrm{H}} 1.56, \mathrm{~s}\right)$ to $\mathrm{C}-3, \mathrm{C}-4, \mathrm{C}-5\left(\delta_{\mathrm{c}} 39.9\right)$, $\mathrm{C}-6\left(\delta_{\mathrm{c}} 25.2\right), \mathrm{C}-7$, and $\mathrm{C}-8$; from $\mathrm{H}_{3}-19\left(\delta_{\mathrm{H}} 1.61, \mathrm{~s}\right)$ to $\mathrm{C}-8\left(\delta_{\mathrm{c}} 136.0\right)$, C-9 $\left(\delta_{\mathrm{c}} 33.7\right), \mathrm{C}-10\left(\delta_{\mathrm{c}} 18.7\right), \mathrm{C}-11\left(\delta_{\mathrm{c}} 31.9\right), \mathrm{C}-12\left(\delta_{\mathrm{c}} 35.2\right), \mathrm{C}-13\left(\delta_{\mathrm{c}}\right.$ $75.0)$, and $\mathrm{C}-14\left(\delta_{\mathrm{c}} 71.9\right)$. The relative configuration of 1 assigned by $2 \mathrm{D}$ 
NOESY spectral data, which indicated the presence of cross peaks between the vinylic $\mathrm{CH}_{3}-18$ and $\mathrm{H}_{2}-6\left(\delta_{\mathrm{H}} 2.32, \mathrm{~m}\right.$ and $\left.2.11, \mathrm{~m}\right)$ suggested the $E$ geometry for the C-3/C-4 double bond, which was also identified by the chemical shift of $\mathrm{H}-3$ at $\delta_{\mathrm{H}} 5.25(\mathrm{~d}, 10.2)$. The geometry of the trisubstituted olefin at C-3/C-4 is $E$ oriented owing to the values of chemical shifts of allylic methylene $\delta_{\mathrm{c}}>30$ and the $J$ values. Cross peaks were assigned between the vinylic $\mathrm{CH}_{3}-19\left(\delta_{\mathrm{H}} 1.61, \mathrm{~s}\right)$ and $\mathrm{H} 2-6(\delta \mathrm{H}$ $1.62, \mathrm{~m}$ and $1.42, \mathrm{~m}, \mathrm{ppm})$ suggested the $\mathrm{E}$ geometry for the $\mathrm{C}-7 / \mathrm{C}-8$ double bond, which was also identified by the chemical shift of $\mathrm{H}-7$ at $\delta_{\mathrm{H}}$ $5.00(\mathrm{~d}, 10.2,5.4)$. The geometry of the trisubstituted olefin at C-7/C-8 was assigned as $E$ based on the higher field chemical shift of the olefinic methyl signal for C-19 $\left(\delta_{\mathrm{C}}\right.$ 17.3). Furthermore, the NOE correlations between $\mathrm{H}-2 / \mathrm{H}-1$, Me-18/H-1 $\left(\delta_{\mathrm{H}} 1.34\right)$, Me-19/ $\mathrm{H}_{2}-10$, Me-20/H-3, Me18/ Me-16, and Me-18/ $\mathrm{H}_{2}-12$, demonstrated the $2 S^{*}, 7 S^{*}$, and $8 S^{*}$ configurations as depicted in Figure 2. A careful analysis of all the NMR spectroscopic data (COESY, HSQC, HMBC, and NOESY) confirmed that (1) is sarcophytolol ${ }^{[7]}$
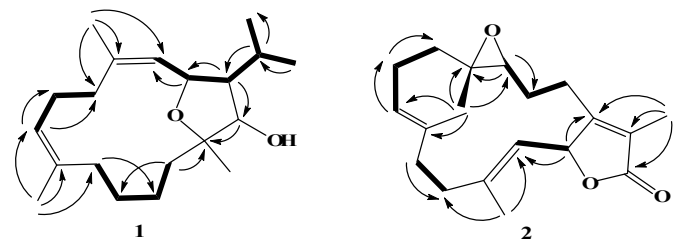

Fig. 2: selected 1H-1H COESY $(\longrightarrow)$ and HMBC ( $\longrightarrow$ ) correlations of compounds 1 and 2

Compound 2 was isolated as faint yellowish oil $(10.0 \mathrm{mg})$ with $[\alpha]$ $\mathrm{D}^{22}-90.0$ (c $0.02, \mathrm{CHCl}_{3}$ ). The LCESI-MS (Negative-ion-mode) of $\mathbf{2}$, exhibited $\mathrm{m} / \mathrm{z} 315.18[\mathrm{M}-\mathrm{H}]^{+}$, consistent with molecular formula $\mathrm{C}_{20} \mathrm{H}_{28} \mathrm{O}_{3}$ (Seven degrees of unsaturation) and validated by HRESI-MS (Positive-ion-mode) $\mathrm{m} / \mathrm{z} 317.2118[\mathrm{M}+\mathrm{H}]^{+}$. The IR spectrum indicated the presence of an $\alpha, \beta$-unsaturated- $\gamma$-lactone $\left(1750 \mathrm{~cm}^{-1}\right)$, an olefin $\left(1669 \mathrm{~cm}^{-1}\right)$, and an epoxide $\left(1256 \mathrm{~cm}^{-1}\right)$ functions. ${ }^{13} \mathrm{C}\left({ }^{1} \mathrm{H}\right.$ decoupled $)$ and DEPT NMR spectra (Table 1) exhibited 20 carbon signals establishing: four methyls, six methylenes, four methines (two are oxygenated), six quaternary carbons, one of which is a carbonyl carbon. Careful examination of the ${ }^{13} \mathrm{C}$ NMR data revealed clear evidences for the presence of the following figures: a) trisubstituted epoxy ring $\delta c 61.4$ $(\mathrm{C}-4, \mathrm{~d})$ and 59.9 (C-5, s) assigned for C-4 and C-5, respectively, b) an $\alpha$, 
$\beta$-unsaturated- $\gamma$-lactone based on characteristic signals resonating at $\delta \mathrm{c}$ $174.0(\mathrm{C}-16, \mathrm{~s}), 120.6(\mathrm{C}-15, \mathrm{~s})$ and 162.2 (C-1, s) supported by IR absorption at $\left.\lambda_{\max } 1750 \mathrm{~cm}^{-1}, \mathrm{c}\right)$ two trisubstituted $\mathrm{C}=\mathrm{C}$ bonds resonating at $\delta \mathrm{c} 124.9(\mathrm{C}-8, \mathrm{~d}), 144.0(\mathrm{C}-9, \mathrm{~s}), 135.6(\mathrm{C}-12, \mathrm{~s})$ and $121.0(\mathrm{C}-13, \mathrm{~d})$ and d) an oxymethine carbon which appeared at $\delta_{\mathrm{C}} 78.8(\mathrm{C}-14)$. The ${ }^{1} \mathrm{H}$ NMR spectrum featured three olefinic methyl protons resonating at $\delta_{\mathrm{H}}$ $1.65\left(\mathrm{CH}_{3}-17, \mathrm{~s}\right), 1.91\left(\mathrm{CH}_{3}-19, \mathrm{~s}\right)$ and $1.88\left(\mathrm{CH}_{3}-20, \mathrm{~s}\right)$; a methyl protons at $1.28\left(\mathrm{CH}_{3}-18, \mathrm{~s}\right)$; two oxymethine protons at $\delta_{\mathrm{H}} 2.68(\mathrm{dd}, 4.8$, $4.2 ; \mathrm{H}-14)$ and at $\delta_{\mathrm{H}} 5.57(\mathrm{dd}, J=10.2,1.2 \mathrm{~Hz} ; \mathrm{H}-14)$ and two olefinic protons at $\delta_{\mathrm{H}} 5.15(\mathrm{dd}, 9.6,5.4 \mathrm{~Hz} ; \mathrm{H}-8)$ and $\delta_{\mathrm{H}} 5.05(\mathrm{dd}, 10.2,1.2 \mathrm{~Hz}$; $\mathrm{H}-13)$. After association of all protons with directly bonded carbons via HSQC spectral measurement, the role of both ${ }^{1} \mathrm{H}-{ }^{1} \mathrm{H}$ COESY and ${ }^{1} \mathrm{H}-{ }^{13} \mathrm{C}$ $\mathrm{HMBC}$ spectra started in structure elucidation. The ${ }^{1} \mathrm{H}-{ }^{1} \mathrm{H}$ COSY spectrum established four proton sequences. The coupling between the methine proton signal resonating at $\delta 2.68(\mathrm{H}-4)$ and the methylenes protons at 1.90 and $1.70(\mathrm{H}-3)$, as well as these signals and the $\mathrm{CH}_{2}$ protons at $\delta 2.40(\mathrm{H}-2)$ established the connectivity of the $\mathrm{H}-4 / \mathrm{H}-3 / \mathrm{H}-2$ fragment (Fig. 2). The second sequence was established from the correlations between the olefinic proton resonating at $\delta 5.15(\mathrm{H}-8)$ and the $\mathrm{CH}_{2}$ protons at 2.13 (H-7), which in turn are correlated with signal of $\mathrm{CH}_{2}$ protons at $\delta 1.10(\mathrm{H}-6)$ confirm the connectivity of the $\mathrm{H}-8 / \mathrm{H}-7 / \mathrm{H}-6$ fragment. The third one was established from the coupling between the $\mathrm{CH}_{2}$ protons resonating at $\delta 2.01$ and $2.20(\mathrm{H}-10)$ with the $\mathrm{CH}_{2}$ protons at 2.78 and $2.10(\mathrm{H}-11)$ led to the connectivity of the $\mathrm{H}-11 / \mathrm{H}-10$ part. Finally, the fourth represents an oxymethine proton $\mathrm{H}-14$ in correlation with an olefinic proton $\mathrm{H}-13$, led to the connectivity of the $\mathrm{H}-14 / \mathrm{H}-13$ fragment. The $\mathrm{HMBC}$ correlations between the methyl protons $\mathrm{H}_{3}-17$ with $\mathrm{C}-16, \mathrm{C}-15$ and $\mathrm{C}-1$ and that of the oxymethine proton $\mathrm{H}-14$ with $\mathrm{C}$ 2 and $\mathrm{C}-13$ led to establishing a trisubstituted furenone fragment. The methyl protons signal at $\delta_{\mathrm{H}} 1.28(\mathrm{~s}, \mathrm{H}-18)$ indicates a proximal oxygen functionality identified from ${ }^{13} \mathrm{C}$ NMR to be an epoxide (Table 1). The location of the epoxide ring at $\mathrm{C} 4 / \mathrm{C}-5$ was detected from $\mathrm{HMBC}$ correlations (Fig. 2), as there are clear correlations between $\mathrm{H}-4$ with $\mathrm{C}$ 3, C-2 and C-5, also correlation between $\mathrm{H}_{3}-18$ with C-5, C-4 and C-6, and between $\mathrm{H}-3$ and $\mathrm{C}-1$ established the connections from $\mathrm{C}-1$ to $\mathrm{C}-6$. The olefinic methyl proton at $\mathrm{H}-19$, also shows an $\mathrm{HMBC}$ correlation with $\mathrm{C}-8, \mathrm{C}-9$ and $\mathrm{C}-10$, while the olefinic proton $\mathrm{H}-8$ is correlated to both $\mathrm{C}-7$ and $\mathrm{C}-9$, prove the connection from $\mathrm{C}-7$ to $\mathrm{C}-10$. The last connection from $\mathrm{C}-11$ to $\mathrm{C}-14$ was concluded from the correlation 
between the olefinic methyl proton $\mathrm{H}-20$ with $\mathrm{C}-11, \mathrm{C}-12$ and $\mathrm{C}-13$. Surveying the soft coral literature especially sarcophyton, led to the assumption of a cembranoid-skeleton backbone. The previous data strongly indicated a cembranoid molecular framework containing position of the epoxide moiety is shifted to be C-4/C-5. The vicinal coupling constant of $10.2 \mathrm{~Hz}$ between $\mathrm{H}-13$ and $\mathrm{H}-14$ as well as 2DNMR NOESY correlation of $\mathrm{H}-14$ with $\mathrm{H}_{3}-20$ established a trans configuration between the $\gamma$-lactone $(\mathrm{H}-14)$ and the olefinic proton $(\mathrm{H}-$ 13). A computer survey was done employing Science Finder Data bases indicated $\mathbf{2}$ is sarcophytolide $\mathrm{B}^{[7]}$.

Compound 3 was obtained as colorless oil $(9.0 \mathrm{mg})$ with $[\alpha]_{\mathrm{D}}^{22}-$ $60.0\left(\mathrm{c} 0.015, \mathrm{CHCl}_{3}\right)$. The negative mode of LCMS exhibited a $[\mathrm{M}-\mathrm{H}]^{+}$ $m / z 315.180$, indicating a molecular formula of $\mathrm{C}_{20} \mathrm{H}_{28} \mathrm{O}_{3}$ and validated by HRESI-MS (Positive-ion-mode) $m / z 317.2118[\mathrm{M}+\mathrm{H}]^{+}$. The seven degrees of unsaturation were supported by NMR data. The IR spectrum indicated the presence of $\alpha, \beta$-unsaturated- $\gamma$-lactone $\left(1750 \mathrm{~cm}^{-1}\right)$, an olefin $\left(1669 \mathrm{~cm}^{-1}\right)$, and an epoxide $\left(1256 \mathrm{~cm}^{-1}\right)$ functions. ${ }^{13} \mathrm{C}$ and DEPT NMR spectral data (Table 1) exhibited 20 carbon signals establishing: four methyls, six methylenes, four methines (including two oxygenated), and six quaternary carbons (including one carbonyl and $\mathrm{sp}^{3}$ carbon). The ${ }^{1} \mathrm{H}$ and ${ }^{13} \mathrm{C}$ NMR spectral data of $\mathbf{3}$ are closely similar to that of $\mathbf{2}$, although during separation process they are quietly differed in the retention factor $\left(\mathrm{R}_{\mathrm{f}}\right)$, which tempted us to check the difference in stereochemistry. 2D NOESY NMR spectral data was employed for assigning the relative configuration of $\mathbf{3}$ (Figure 2). The presence of a NOESY cross peak between the vinylic $\mathrm{H}-8$ and $\mathrm{H}-13$, cross peaks between $\mathrm{CH}_{3}-20$ and $\mathrm{H}-12, \mathrm{CH}_{3}-19$ and $\mathrm{H}-11$, indicated the $Z$ geometry of both $\mathrm{C}-13 / \mathrm{C}-14$ and $\mathrm{C}-9 / \mathrm{C}-10$ double bonds. This deduction supported by the chemical shift of $\mathrm{H}-14$ at $\delta_{\mathrm{H}} 4.99$ (dd, 9.6, 1.2). Furthermore, the conclusive NOE correlations between $\mathrm{H}-14$ and $\mathrm{H}-1$; between $\mathrm{H}-14$ and $\mathrm{CH}_{3}-20$; between $\mathrm{H}-3$ and $\mathrm{H}-$, confirmed the $Z$ geometry of the two vinylic double bonds. The absence of cross peaks between $\mathrm{CH}_{3}-18$ and $\mathrm{H}-5$ indicated the trans configuration. After survey employing different data bases includes Science Finder, indicates 3 is sarcophytolide $C^{[7]}$.

Cytotoxicity of the isolated compounds (1-6) was evaluated employing five cancer panels; HepG2, MCF-7, A549, PC-3 and VERO (Table 2). 5-fluorouracil was used as a positive control. Interestingly, compounds (1 and $\mathbf{3}$ ) exhibited selective cytotoxic effects on HepG2 and showed no toxicity on the other cell lines. However, 2 showed selective 
cytotoxic effect on MCF-7 with no toxicity on other cell lines. Meanwhile, 4-6 showed cytotoxic activity against both MCF-7 and HepG2 with no toxicity on normal Vero cells.

Table 2. Cytotoxic activity of the compounds 1-6, towards five cancer cell lines

\begin{tabular}{cccccc}
\hline Comp. No. & \multicolumn{5}{c}{ IC $_{\mathbf{5 0}}(\mu \mathrm{g} / \mathrm{mL})$} \\
\cline { 2 - 5 } & $\mathbf{H p G 2}^{\mathrm{a}}$ & MCF-7 & A549 & PC-3 & VERO \\
\hline $\mathbf{1}$ & $20 \pm 0.032$ & $\mathrm{ND}^{\mathrm{b}}$ & $196.7 \pm 0.032$ & $31.5 \pm 0.032$ & $\mathrm{ND}$ \\
& & & & & \\
$\mathbf{2}$ & $\mathrm{NT}$ & $25 \pm 0.164$ & $73.8 \pm 0.042$ & $216.5 \pm 0.164$ & $\mathrm{ND}$ \\
& & & & & \\
$\mathbf{3}$ & $20 \pm 0.153$ & $\mathrm{NT}$ & $200.7 \pm 0.164$ & $112.4 \pm 0.032$ & $\mathrm{ND}$ \\
& & & & & \\
$\mathbf{4}$ & $20 \pm 0.068$ & $20 \pm 0.054$ & $98.4 \pm 0.120$ & $09.3 \pm 0.164$ & $\mathrm{ND}$ \\
& & & & & \\
$\mathbf{5}$ & $25 \pm 0.089$ & $30 \pm 0.131$ & $88.4 \pm 0.135$ & $155.3 \pm 0.171$ & $\mathrm{ND}$ \\
$\mathbf{6}$ & $26 \pm 0.061$ & $33 \pm 0.075$ & $100.4 \pm 0.092$ & $17.3 \pm 0.125$ & $\mathrm{ND}$ \\
\hline
\end{tabular}

${ }^{\mathrm{a}}$ Human hepatocellular liver carcinoma (HepG2), human breast adenocarcinoma (MCF-7),

Lung carcinoma (A549) and normal adult African green monkey kidney (VERO). Prostate cancer (PC-3)

${ }^{\mathrm{b}} \mathrm{ND}$ : not detected.

\section{Acknowledgment}

Grateful to King Abdulaziz university, Jeddah, Saudi Arabia, for supporting this work.

\section{References}

[1] Duffy, R. and Wade, C.; Discovery of anticancer drugs from antimalarial natural products, Drug Discovery Today, 17, $942-953$ (2012).

[2] Kamb, A., Wee, S. and Lengauer, C., Why is cancer drug discovery so difficult, Nat. Rev. Drug Discov., 6, 115-120 (2007).

[3] Newman, D. J., and Cragg, G. M.; Natural Products as Sources of New Drugs over the Last 25 Years, J. Nat. Prod., 70, 461-477 (2007).

[4] Burgess, J. G.; New and emerging analytical techniques for marine biotechnology, Curr. Opin. Biotechnol., 23, 29-33 (2012).

[5] Edwards, A. J. and Head, S. M.; Key Environments-Red Sea; Pergamon Press: Oxford, UK, (1987), p. 440.

[6] Roethle, P. A. and Trauner, D.; The cheistry of marine furanocembranoids, pseudopteranes, gersolanes, and related natural products, Nat. Prod. Rep., 25, 298-317 (2008).

[7]. Al-lihaibi, S. S., Alarif, W. M., Abdel-lateff, A., Ayyad, S.-E. N., Abdel-Naim, A. B., ElSenduny, F. F. and Badria, A. A.; Three new cembranoid-type diterpenes from Red Sea soft coral Sarcophyton glaucum: isolation and Antiproliferative activity against HepG2 cells, European Journal of Medicinal Chemistry, 81, 314-322 (2014). 


\title{
أيزوبرينات سامة من مراجين البحر الاحمر ساركوفيتون جلوكوم و زينيا أمبيلاتا
}

\author{
إلهام /حمد سلم، خالد عمر الفوتى، و سيف الدين نصر عباد \\ قسم الكبيباء، كلية العلوم، جامعة الملك عبد العزبز، جدةـ المدلكة العربية السعودية \\ E-mail: snayyad2@yahoo.com
}

الستخلص. ثلاثة سيمبرانويدات سامة هم: ساركوفيتولول (1) و ساركوفيتوليد ب (2) و ساركوفيتوليد سى (3) تم تعريفهم من الجزء الذائب فى الاثير ثنائى إيثيل من المستخلص العضوى للمرجان الناعم من نوع ساركوفيتون جلوكوم و مصدره ساحل البحر الاحمر السعودى، بالاضافة الى ثلاثة سسكوتربينات من لاني

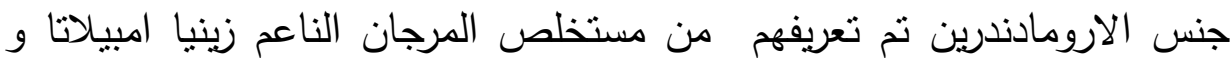
هي: اروماديندرين (4) و بالوسترول (5) و فيريديفلورول (6). نم إثبات التركيب الكيميائى باستخدام الطرق الطيفية المختلفة منل الرنين النووى المغناطيسى و طيف الاشعة فوق البنفسية و تحت الحمراء بالإضافة الى مطياف الكتلة كما تم إجراء اختبار قياس السمية على المركبات المفصولة كمضادات لسرطان الكبد وسرطان الثثي و سرطان الرئة و سرطان البروستاتا و كذللك سرطان كلى القرد الإفريقيي الناضج الأخضر • ووجد أن المركبين رقم 1 و 3 لهما سمية اختيارية الرية نحو سرطان الكبد و مركب رقم 2 له سمية اختبارية نحو سرطان الثدي كما وجد ان المركبات رقم -6 و 4 لها سمية اختبارية لسرطان الكبد وسرطان الثثي. 